\title{
Ciclo de Mejora Docente en el Aula aplicado a la asignatura de Prehistoria I: \\ Las Sociedades Cazadoras y Recolectoras. Grado en Historia (Universidad de Sevilla)
}

\section{Teaching Improvement Cycle applied to the subject of Prehistory I: The Hunter and Collector Societies. Degree in History (University of Seville)}

MARÍA Dolores Simón-VALLEjo

ORCID: https://orcid.org/0000-0002-6885-1464 Universidad de Sevilla

Departamento de Prehistoria y Arqueología msimon@us.es

Fecha de recepción:

Fecha de aceptación:

DOI: http://dx.doi.org/10.12795/9788447221912.118 Pp.: 2613-2630 


\section{Resumen}

En la presente publicación se detalla el Ciclo de Mejora en el Aula aplicado al tema 4. Origen y evolución del hombre, de la asignatura Prehistoria I, obligatoria de primer curso del Grado en Historia de la Universidad de Sevilla durante el curso académico 2019-2020. La duración del CIMA fue de ocho horas.

Palabras clave: Prehistoria I, Experimentación docente, Docencia Universitaria.

\section{Abstract}

This publication details the Teaching Improvement Cycle applied to topic 4. Origin and Human evolution, prehistory I, compulsory first year of the Degree in History of the University of Seville during the academic year 2019-2020. The cycle duration is scheduled for eight hours.

Key Words: Prehistory I, Teaching Experimentation, University Teach

Jornadas de Formación e Innovación Docente del Profesorado | № 2 (2019)

Esta obra se distribuye con la licencia Creative Commons 


\section{Introducción}

El Ciclo de Mejora Docente en el Aula (de ahora en adelante, CIMA) ha sido aplicado en la asignatura: Prehistoria I. Las Sociedades cazadoras y recolectoras que se imparte en la Facultad de Geografía e Historia y pertenece al Grado de Historia, es obligatoria de primer curso y se impartió en el primer cuatrimestre.

El alumnado que cursa esta asignatura está compuesto principalmente por discentes que han terminado el año anterior Bachillerato y por tanto es el primer contacto con un aula universitaria. También hay que contemplar que la clase está compuesta por un alumnado minoritario de edad madura (con una edad que oscila entre 45 - 70 años), así como un número reducido de repetidores. (en total 5 alumnos). En conjunto, de los 69 matriculados, participan habitualmente 50 alumnos.

El CIMA de ocho horas, fue aplicado en el bloque III. Grupos Culturales del Pleistoceno. $Y$ en concreto en el tema 4. Origen y evolución del hombre. Dispersión mundial. Durante el curso académico 2019-2020.

Dada la extensión del tema a tratar, la segunda parte, dispersión mundial de los distintos homínidos, no formó parte de los contenidos ni de las actividades a desarrollar durante la experimental.

\section{Diseño previo del Ciclo de Mejora en el Aula.}

El CIMA consta de varios apartados, una primera formulación de nuestro Modelo metodológico posible, un cuestionario inicial, el diseño del Mapa de contenidos y por último la secuencia de actividades programadas.

Jornadas de Formación e Innovación Docente del Profesorado | № 2 (2019)

Esta obra se distribuye con la licencia Creative Commons 


\section{Modelo metodológico posible}

Durante el curso de docencia universitaria he tenido la ocasión de reflexionar sobre mi modelo metodológico personal y, tras la lectura de algunos capítulos de la obra de Bain, K. (2007), pude extraer algunos principios fundamentales que han enriquecido y/o fortalecido mi modelo metodológico. Estos principios podrían sintetizarse en lo siguiente:

- Crear un entorno crítico natural, consistente en despertar la curiosidad desde la razón crítica (duda cartesiana). Este ambiente se puede crear con diversas estrategias: desafios, preguntas, intriga... y, todo ello, genera un auténtico motor del aprendizaje.

- Planteamiento de Pregunta/s y/o Problema/s a los alumnos.

- Orientaciones de ayuda al estudiante para que comprenda lo planteado y motivarlo para que lo solucione.

- Participación activa de los alumnos como forma de construir su compresión del problema. Los estudiantes como protagonistas de la clase y responsables de su aprendizaje se hacen preguntas y están preparados para sacar conclusiones e ideas claves/básicas.

- Cambio del papel del profesor como orientador del proceso.

- Comenzar con los estudiantes en lugar de con la disciplina.

- Considerar de donde parten los estudiantes e introducir actividades prácticas que vayan de lo simple a lo complejo. Los cuestionarios iniciales permiten conocer las ideas iniciales de los alumnos.

- Es necesario establecer compromisos con los alumnos, para que asuman la responsabilidad del propio aprendizaje y adquieran conductas individuales y colectivas como ser puntual, participativo, etc. Es preciso que el profesor cree en la clase un ambiente idóneo para entablar un dialogo productivo.

Jornadas de Formación e Innovación Docente del Profesorado | № 2 (2019)

Esta obra se distribuye con la licencia Creative Commons 
- Los profesores deben ayudar a los alumnos a aprender de manera comprensiva en lugar de memorizar, promoviendo un "razonamiento disciplinar"

- Huir de la monotonía, creando diversidad en las formas de presentar la información y de secuenciar la manera de aprender.

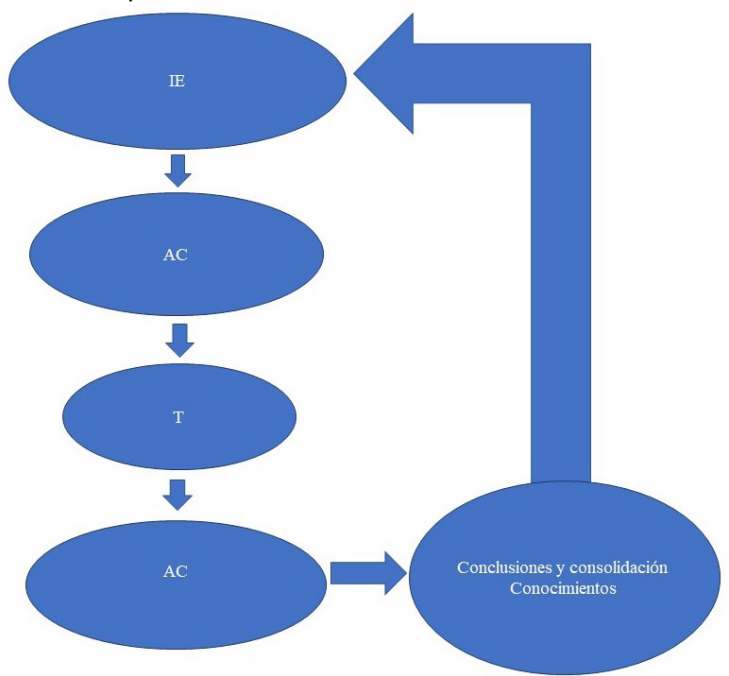

Figura 1. Modelo Metodológico Posible. IE. Ideas de las que parten los alumnos. AC. Actividades de Contraste. T. Teoría interactiva. AC. Actividades de Contraste. Conclusiones y consolidación de los conocimientos adquiridos.

\section{Mapa de Contenidos del Ciclo de Mejora Docente en el Aula}

El tema que ocupará este CIMA versará sobre el origen del hombre. Dado que es un tema delicado por las implicaciones de género que conlleva, tengo especial cuidado en el vocabulario que utilizo e introduzco un lenguaje más inclusivo, pues es muy común y generalizado hablar del origen del hombre en lugar del origen de la humanidad o de los humanos.

El Mapa ha sido diseñado teniendo en cuenta distintos tipos de contenidos: teóricos (C1), procedimentales psicomotrices (P), datos (D), actitudes y valores ( $V$ ) y

Jornadas de Formación e Innovación Docente del Profesorado | № 2 (2019)

Esta obra se distribuye con la licencia Creative Commons 
procedimentales intelectuales (C2). C1: Racionalizar la evolución; Identificar rasgos de lo humano y diferenciarlos con respecto a otros primates. C2: Comparar, analizar, argumentar, identificar y descubrir; Elaborar mapas de situación, conclusiones. V: Valorar nuestro pasado común; Sensibilizar sobre aspectos actuales, igualdad de género, respeto a las diferencias; Valorar el patrimonio geográfico. P: Situarse en un mapa geográfico, habilidades de situación espacial. D: Datos geográficos y cronológicos, vocabulario específico.

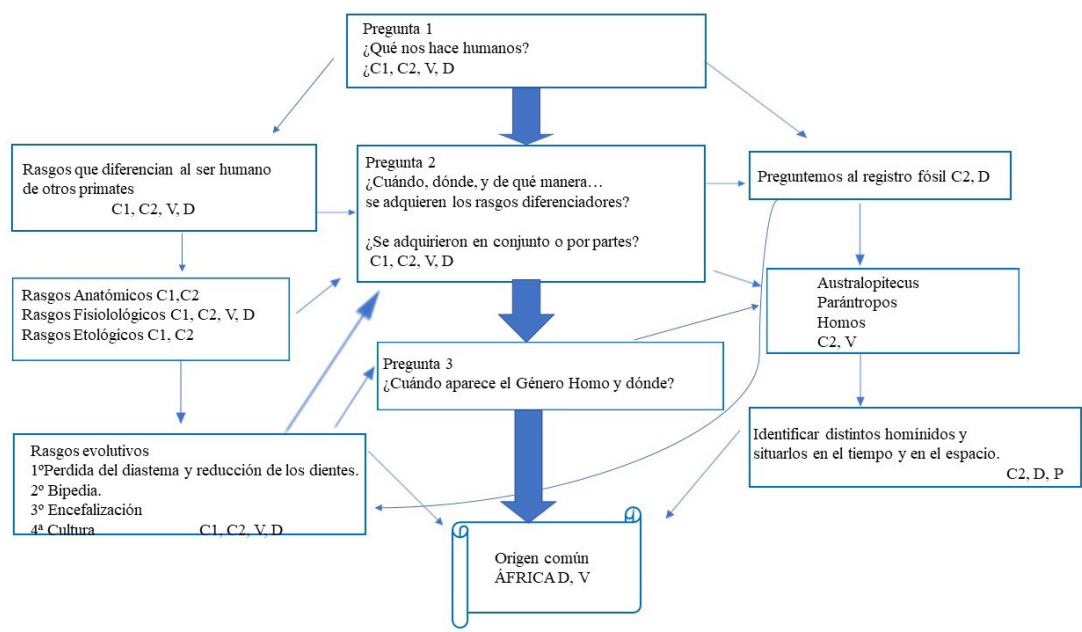

Figura 2. Esquema del Mapa de Contenidos y Preguntas clave.

\section{Cuestionario inicial}

Con carácter previo a la realización del CIMA realizamos un cuestionario inicial a los alumnos con la finalidad de conocer los conocimientos, errores e ideas previas, con las que partiríamos y con las que tendríamos que trabajar nuestra secuencia de actividades.

Las preguntas formuladas en el cuestionario inicial fueron las siguientes:

1. ¿Que nos hace humanos?

2. ¿Cuál es nuestro origen?

Jornadas de Formación e Innovación Docente del Profesorado | № 2 (2019)

Esta obra se distribuye con la licencia Creative Commons 


\section{Secuencia de actividades programadas}

Tengo por costumbre dejar 5 de cortesía a la entrada y a la salida, detalle que los alumnos agradecen bastante, considero importante crear un ambiente cordial, que facilite el trabajo posterior en clase. En consecuencia, las clases son de 50 minutos.

En el CIMA voy a poner power point interactivos. En ellos hay conceptos, imágenes, mapas, citas bibliográficas, preguntas que incitan al razonamiento crítico, esquemas que incentiven la curiosidad inherente a cualquier proceso científico, también introduciré elementos sorpresa, que en muchas ocasiones son elementos con los que los alumnos están familiarizados, como videojuegos, películas, situaciones de la vida diaria, etc.

Tabla 1. Secuencia de actividades del Ciclo de Mejora en el Aula. 1a Sesión.

1a Sesión. Pregunta 1. ¿Que nos hace humanos? ¿Cómo identificamos lo humano?

\begin{tabular}{|c|c|c|}
\hline Actividad & Descripción & Tiempo \\
\hline $\begin{array}{l}\text { Cuestionario inicial. } \\
\text { Punto de partida errores y } \\
\text { aciertos de los alumnos }\end{array}$ & $\begin{array}{l}\text { Ya hemos realizado en una sesión anterior } \\
\text { el cuestionario inicial y lo tenemos } \\
\text { analizado, por tanto, conocemos los errores } \\
\text { de los que parten nuestros alumnos. }\end{array}$ & $30 \mathrm{~m}$ \\
\hline $\begin{array}{l}\text { Ideas-errores de los } \\
\text { alumnos }\end{array}$ & $\begin{array}{l}\text { Parto de las ideas de los alumnos } \\
\text { expresadas en el cuestionario inicial }\end{array}$ & \\
\hline $\begin{array}{l}\text { Actividades de contraste. } \\
\text { Pregunta clave } \\
\text { acompañada de } \\
\text { lluvia de preguntas/ } \\
\text { respuestas de los } \\
\text { alumnos. } 15 \text { minutos. } \\
\text { Recitamos en clase } \\
\text { nuestro "nombre primate", } \\
\text { nos identificamos con } \\
\text { una denominación } \\
\text { taxonómica. }\end{array}$ & $\begin{array}{l}\text { Comenzamos con la pregunta ¿Quiénes } \\
\text { somos?, cuestión que ya nos hicimos el } \\
\text { primer día de clase y recuerdo que es una } \\
\text { de las preguntas clave de la asignatura. } \\
\text { Una vez hemos terminado esta lluvia de } \\
\text { preguntas, les invito a recitar en clase } \\
\text { su "nombre primate" (denominación } \\
\text { taxonómica del ser humano). } \\
\text { "Somos primates, antropoideos, catarrinos, } \\
\text { hominoideos, homínidos y homo sapiens } \\
\text { sapiens". }\end{array}$ & $15 \mathrm{~m}$. \\
\hline
\end{tabular}

Jornadas de Formación e Innovación Docente del Profesorado | № 2 (2019)

Esta obra se distribuye con la licencia Creative Commons 
Tabla 3. Secuencia de actividades del Ciclo de Mejora en el Aula. 3a Sesión.

\section{3a Sesión}

Problema 3. ¿Cuándo aparece el género Homo? y dónde.

\begin{tabular}{|l|l|l|}
\hline Actividad & Descripción & Tiempo \\
\hline contraste & $\begin{array}{l}\text { Vuelvo a llevar la cuerda del tiempo para situarnos en } \\
\text { el momento donde aparece el Género Homo. También } \\
\text { nos servirá para situarnos en el temario temporal de } \\
\text { la asignatura, recordaremos y haremos una puesta en } \\
\text { común de todas las preguntas y respuestas del tema } \\
\text { hasta el momento. }\end{array}$ & \\
& $\begin{array}{l}\text { Recuerdo que esta actividad consiste en explicar } \\
\text { el tiempo en la Prehistoria con una cuerda de 20 } \\
\text { metros, que representa los 6 millones de años de }\end{array}$ & \\
& $\begin{array}{l}\text { Prehistoria. Realizo la proporción hasta llegar al inicio } \\
\text { de la Historia (5000 años) = 1.5 cm. de modo que el } \\
\text { descubrimiento de América ocupa en la cuerda menos } \\
\text { de un centímetro. A lo largo de la cuerda coloco cinta } \\
\text { adhesiva de colores con algunos hitos importantes } \\
\text { dentro de la evolución humana, primeros homínidos, } \\
\text { bipedia, fuego, primeros agricultores y ganaderos, }\end{array}$ & \\
& invención de la escritura, descubrimiento de América. & \\
\hline
\end{tabular}

Jornadas de Formación e Innovación Docente del Profesorado | № 2 (2019)

Esta obra se distribuye con la licencia Creative Commons 


\begin{tabular}{|l|l|l|}
\hline Teoría & $\begin{array}{l}\text { Teoría con power point interactivo. } \\
\text {-Distintos homínidos del Género Homo. } \\
\text { Se introducen preguntas, mapas de situación de los } \\
\text { distintos homínidos, problemática sobre la adscripción } \\
\text { u homínidos precedentes. Coexistencia de distintas } \\
\text { especies. Todo se presenta de forma que inciten al } \\
\text { razonamiento crítico y que incentiven la curiosidad. }\end{array}$ & \\
\hline
\end{tabular}

Tabla 4. Secuencia de actividades del Ciclo de Mejora en el Aula. 4a Sesión.

\begin{tabular}{|c|c|c|}
\hline Actividad & Descripción & Tiempo \\
\hline $\begin{array}{l}\text { Cuestionario final y } \\
\text { conclusiones. } \\
\text { Puesta en común. }\end{array}$ & $\begin{array}{l}\text { Se vuelve a dar a los alumnos los cuestionarios y } \\
\text { vuelven a contestar. } \\
\text { Reevaluación de los conceptos vistos. cuestionario } \\
\text { inicial y su contrastación con lo que respondieron } \\
\text { en el inicio. } \\
\text { Conclusiones finales del tema, por parte de los } \\
\text { alumnos. }\end{array}$ & $30 \mathrm{~m}$. \\
\hline
\end{tabular}

\section{Aplicación del CIMA. Diario de las sesiones}

Antes de comenzar, tengo que hacer una reflexión sobre el medio físico donde imparto las clases, el tipo es de "Aula Magna", asientos escalonados para el alumnado y espacio del profesor elevado y con una gran mesa reservada para él.

Durante el transcurso de las clases tan solo ocupo el puesto del profesor para firmar y hacer usos del ordenador y la pantalla. El resto del tiempo estoy de pie, acercándome a los alumnos, de esta manera aminoro los condicionantes físicos del medio, fracturando el binomio espectador/pasivo y profesor/activo.

Jornadas de Formación e Innovación Docente del Profesorado | № 2 (2019)

Esta obra se distribuye con la licencia Creative Commons 
Durante el desarrollo del CIMA, ya no estaban tan nerviosos y expectantes como a comienzo de curso y cuando presenté el cuestionario inicial, se mostraron bastante participativos, les gustó la propuesta y la realizaron con bastante interés.

\section{Diario de las sesiones}

\section{1ạ sesión.}

Durante la actividad de contraste "nombre primate" (denominación taxonómica), y aunque la denominación es bastante formalizada y académica, les encantó recitarlo en clase e incluso lo memorizaron (en las siguientes sesiones lo he podido comprobar). Se sintieron iguales entre sí, al recitar el nombre, unos alumnos comentaron que nunca habían imaginado que tenían ese nuevo nombre, con tantos apellidos. Acto seguido, sin incitarlos comenzaron a preguntar que significaban todas las denominaciones, esto excedió el tiempo programado de 15 minutos.

La teoría no fue tal, pues se suscitó un encadenamiento de preguntas, que desbordaron el tiempo asignado, de modo que, cuando más animada estaba la clase, se acabó el tiempo (restringido a una hora). No pude terminar la sesión en el tiempo programado.

2o- Sesión.

Tras el video la evolución en un minuto, se quedaron muy impactados y me pidieron volverlo a ver. Acto seguido comenzaron a trabajar en grupo, hubo algo de dificultad durante el trabajo con los mapas geográficos, pues yo pensaba que sabrían leerlos y tuve que emplear tiempo extra en enseñarles, esto retrasó el desarrollo de esta fase.

Durante la teoría y en el tema de la bipedia, no se entendían ciertos aspectos de antropología fisica y, aunque no estaba en la programación, y dado que mi despacho

Jornadas de Formación e Innovación Docente del Profesorado | № 2 (2019)

Esta obra se distribuye con la licencia Creative Commons 
está junto al aula, llevé a clase un esqueleto humano, cosa que agradecieron, pues así pudimos ver mejor algunos aspectos antropológicos. Con anterioridad no había considerado esta posibilidad, pues me parecía demasiado complejo.

Durante la práctica interactiva el coleccionista de cerebros, se suscitaron y encadenaron tantos temas y preguntas, que excedió el tiempo programado, introduje teoría a petición de los alumnos. La participación y entusiasmo del alumnado fue muy gratificante. La práctica de bipedia y evolución no la he impartido como tal, pues quedó diluida e inserta en la teoría que pidieron los alumnos, que incluso hablaban entre ellos y discutieron temas específicos. En una ocasión invité a que dos alumnos nos contaran aquello que tan acaloradamente estaban hablando y que tenían relación con el tema de evolución que estábamos viendo en clase. En ese momento, se me ocurrió desarrollar, para el próximo año, una actividad de contraste a partir de estas "micro charlas" o "micro congresos", y que las llevaran a cabo entre 2-3 alumnos y que una vez terminaran, se levantarán y buscaran entre el resto de la clase, alumnos pros y contra de sus argumentos, actividad que se podría continuar para las siguientes horas de clase.

Durante la lectura del texto, ya comentaron por sí mismos que el texto era del siglo XIX y argumentaron que un historiador debía de tener razonamiento crítico para analizar las fuentes escritas. Planteamiento que me parece muy satisfactorio, pues a lo que estoy acostumbrada con alumnos de primero, es a un silencio en clase o a no ofrecer explicaciones como esta.

3osesión.

Cuando llevábamos realizadas 5 horas del CIMA, en un momento de confidencias en clase, me han comentado entre todos que tenían mucho trabajo que entregar en otras asignaturas e incluso pequeños exámenes, pero que era una de las pocas clases que no se perdían porque estaban "enganchados" al tema, no se querían perder de que

Jornadas de Formación e Innovación Docente del Profesorado | № 2 (2019)

Esta obra se distribuye con la licencia Creative Commons 
trataríamos al día siguiente. Creo que he conseguido despertar la curiosidad y el interés por el tema. En la participación también se nota, de un total de 69 matriculados, asisten con asiduidad a clase unos 50, y todo a pesar de no pasar lista de firma, ni controlar la asistencia, ni de incentivarla de forma coercitiva.

\section{Evaluación del aprendizaje de los estudiantes. Análisis comparativo de los cuestionarios inicial y final. Escaleras de aprendizaje}

Una de las opciones posible para conocer y analizar los modelos de los estudiantes, son los cuestionarios, estos nos han permitido recoger respuestas por escrito de los alumnos en su conjunto y realizar un análisis sistemático de los niveles de conocimientos, antes de comenzar el CIMA (Porlán, 2017).

Sobre 50 cuestionarios recogidos, hemos seleccionado una muestra de 20, sobre estos hemos realizado el estudio de escaleras de aprendizaje que mostramos en la figura 3. En ellas podemos observar la evolución de los alumnos, antes y después de la aplicación del CIMA. Los distintos modelos de pensamiento de los alumnos desde el principio del tema hasta el estadio final han mejorado considerablemente, observándose un incremento cualitativo en las respuestas.

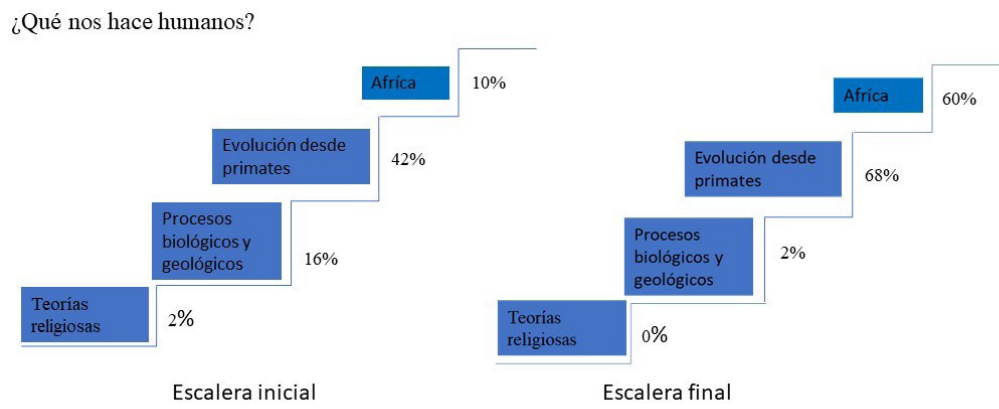

№ alumnos $=20$

Figura 3. Escaleras de aprendizaje correspondientes a la primera pregunta del cuestionario inicial y final

Jornadas de Formación e Innovación Docente del Profesorado | № 2 (2019)

Esta obra se distribuye con la licencia Creative Commons 
¿Cuál es nuestro origen?

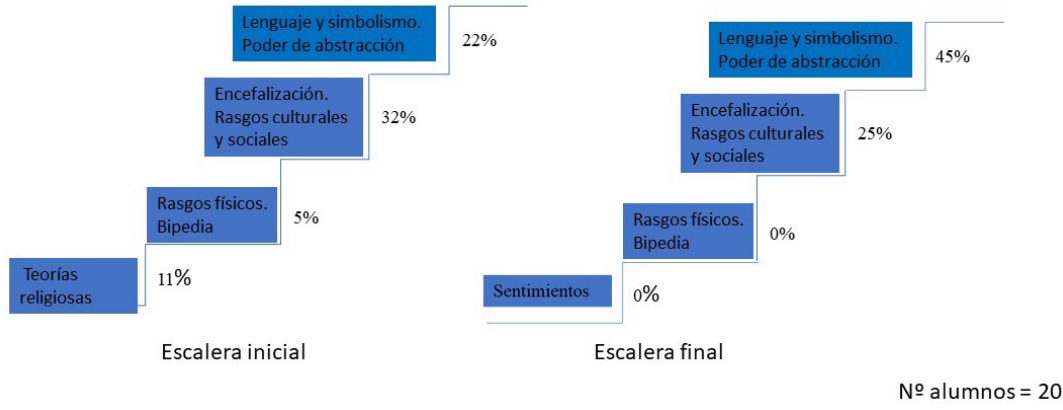

Figura 4. Escaleras de aprendizaje correspondientes a la segunda pregunta del cuestionario inicial y final

Tras el análisis de los cuestionarios, los alumnos han alcanzado han superado los primeros obstáculos y en general, mas del 50\% se encuentran en los niveles de aprendizaje avanzados, el hecho de que no todos hallan alcanzado un el cuarto "escalón" se debe principalmente a la duración del tema que es más extenso que la duración del CIMA.

\section{Evaluación del CIMA}

¿Qué ha ido bien durante el Ciclo de Mejora en el Aula? La alta participación, el interés y la motivación de los alumnos. Me ha gratificado bastante la emoción que he observado en más del 70\% del alumnado. Han funcionado muy bien las propuestas de utilización de un lenguaje inclusivo cuando hablamos de evolución humana. En este sentido, parte de las alumnas promovieron la iniciativa de emplear siempre este tipo de términos más acordes con la igualdad de género. El resto de los alumnos estuvieron de acuerdo con esta propuesta.

Han funcionado muy bien los power point interactivos o de teoría interactiva, que no se corresponden con los apuntes tradicionales de dictar y copiar. En ellos hay conceptos, preguntas que incitan al razonamiento crítico,

Jornadas de Formación e Innovación Docente del Profesorado | № 2 (2019)

Esta obra se distribuye con la licencia Creative Commons 
mapas, imágenes, esquemas que incentivan la curiosidad inherente a cualquier proceso científico, también introduzco elementos sorpresas, que en muchas ocasiones son elementos de la vida cotidiana con los que los alumnos están familiarizados, como videojuegos, películas y eventos que tienen lugar durante el desarrollo de las clases, como por ejemplo Halloween, etc.

¿Qué creo que no ha funcionado? El tiempo programado ha sido insuficiente, he necesitado más tiempo del previsto. En algunos momentos no me esperaba que las sesiones fuesen tan dinámicas. En los trabajos en grupo, eran demasiados alumnos, y se ha empleado mucho tiempo. Además, el aula fisica ha supuesto un cierto condicionante, dado que no está pensada para hacer este tipo de actividades en grupo.

¿Qué cambios realizaría para el próximo año? Tener en cuenta la realización de las tareas y que tengan alguna gratificación en la calificación final. Pasar un listado de firmas y tener más control de los trabajos. Realizar el cuestionario inicial con tiempo para conocer los resultados de las escaleras de aprendizaje y, de esta manera, programar mejor las actividades de contraste y planificar bien los tiempos. En la programación que realicé me he quedado muy por encima de las 8 horas previstas, pues he necesitado 12 horas para poder completar el CIMA completo.

\section{Reflexión final sobre la experiencia del CIMA y adopción a la docencia habitual de Principios Didácticos.}

En general, estoy bastante satisfecha con el Ciclo de Mejora en el Aula aplicado. Me ha sorprendido la alta participación del grupo y el interés mostrado. El ambiente ha sido muy bueno, con un alto grado de interés y motivación.

Jornadas de Formación e Innovación Docente del Profesorado | № 2 (2019)

Esta obra se distribuye con la licencia Creative Commons 
Desde la perspectiva proporcionada por todo el trabajo y teniendo en cuenta los principios que proponen distintos autores (Bain, 2007; Finkel, 2008; Porlán (coord.), 2017), estos serán algunos de los Principios Didácticos que adoptaremos en nuestros futuros Ciclos de Mejora Experimentales en el Aula. (según Porlán).

1. Elaborar cuestionarios para conocer los modelos mentales e hipótesis de los estudiantes sobre los problemas al inicio y al final de un periodo de clases.

2. Diseñar actividades de contraste que cuestionen las hipótesis de los estudiantes y les ayuden a superar los obstáculos.

3. Diseñar y aplicar secuencias de actividades coherentes con el modelo metodológico posible.

4. Formular contenidos de aprendizaje de diversos tipos para promover una formación integral de los estudiantes.

5. Seleccionar los contenidos más organizadores y establecer las relaciones más significativas entre ellos mediante la elaboración de mapas.

6. Formular problemas o preguntas claves vinculados a los contenidos, a las secuencias de actividades y a los cuestionarios.

7. Elaborar y reformular, a partir de la experiencia, el modelo metodológico ideal y posible.

Jornadas de Formación e Innovación Docente del Profesorado | № 2 (2019)

Esta obra se distribuye con la licencia Creative Commons 


\section{Referencias bibliográficas}

Bain, K. (2007). Lo que hacen los mejores profesores universitarios. Valencia, Publicaciones de la Universidad de Valencia.

Finkel, D. (2008). Dar clase con la boca cerrada. Valencia, Publicaciones Universidad de

Valencia.

Porlán, R. (Coord.) (2017). Enseñanza universitaria. Cómo mejorarla. Madrid, Ed. Morata.

Jornadas de Formación e Innovación Docente del Profesorado | № 2 (2019) 